\title{
In the Zone: lessons from the first Canadian emergency department application of resuscitative endovascular balloon occlusion of the aorta (REBOA)
}

\author{
Markus T. Ziesmann, MD*†; Joao Rezende-Neto, MD, PhD**; Katherine McKendy, MD, MEd ${ }^{*}$; \\ Vikramaditya Prabhudesai, MBBS, MS${ }^{\S}$; Sandro Rizoli, MD, PhD ${ }^{* \dagger ;}$; Andrew Petrosoniak, MD, MSc ${ }^{\mathbb{I} \|}$
}

Keywords: hemorrhagic shock, REBOA, trauma

\section{INTRODUCTION}

Resuscitative endovascular balloon occlusion of the aorta (REBOA) is a technique for managing non-compressible hemorrhagic shock. A recent report combined the experience of 11 hospitals performing REBOA; however, none of the sites were located in Canada. ${ }^{1}$ At our institution, while we have used REBOA within the operating room (OR), herein, we describe the first deployment of this technology in a Canadian emergency department (ED). ${ }^{2}$

\section{CASE REPORT}

A previously healthy, 52-year-old male in hemorrhagic shock, from a high-speed, side-impact motor vehicle collision at 06:50, underwent initial resuscitation at a non-trauma centre, including intubation and a massive blood-based resuscitation. Upon arrival, the patient was profoundly hypotensive (blood pressure [BP] 55/ 30) and Glasgow Coma Scale (GCS) of 8 (eyes 1, verbal 2 , and motor 4). Basic investigations included a chest $\mathrm{X}$-ray and pelvis $\mathrm{X}$-ray, demonstrating bilateral pneumothoraces and an unstable open-book pelvis fracture (anteroposterior compression II, Young Burgess
Classification). Management prior to transfer included insertion of bilateral chest tubes, application of a pelvic binder, and administration of 1 gram of tranexamic acid (TXA). He received 4 litres of $0.9 \%$ normal saline, 10 units of packed red blood cells (pRBCs), and 1 unit of fresh frozen plasma (FFP). Despite this massive resuscitation, an epinephrine infusion $(1 \mathrm{mcg} / \mathrm{kg} / \mathrm{min})$ was initiated prior to transfer to our trauma centre because of persistent hypotension (BP 60/40).

The patient was received at our trauma centre at 09:50, under the care of a Royal College emergency medicine (FRCP-EM)-trained trauma team leader (TTL) in charge of a multi-disciplinary trauma team including anesthesia and orthopedic residents, a trauma surgery resident and fellow, a staff trauma surgeon, three trauma nurses, and two respiratory therapists. On repeat assessment, the airway remained secure, and an X-ray demonstrated bilateral lung re-expansion. A FAST exam was positive in the abdominal views, and the pelvis, previously bound, was not re-examined. During transfer, the patient had required the addition of a norepinephrine infusion; both norepinephrine and epinephrine infusions were dosed at $1 \mathrm{mcg} / \mathrm{kg} / \mathrm{min}$, with the systolic blood pressure (sBP) remaining between 45 and $60 \mathrm{~mm} \mathrm{Hg}$, heart rate of $102 \mathrm{bpm}$, and temperature of $33.2^{\circ} \mathrm{C}\left(91.8^{\circ} \mathrm{F}\right)$. Initial investigations included a $\mathrm{pH}$ of $<6.8$ (normal 7.35-7.42), lactate of $>15.0 \mathrm{mmol} / \mathrm{L}$ (normal 0.5-2.3), fibrinogen $0.5 \mathrm{~g} / \mathrm{L}$ (normal 1.8-4.0), hemoglobin of $143 \mathrm{~g} / \mathrm{L}$ (normal 130-170), and platelets of $106 \times 10^{9} / \mathrm{L}$ (normal 140-400).

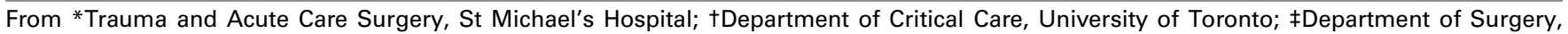
University of Toronto; §Department of Medical Imaging, St. Michael's Hospital, University of Toronto; ๆDepartment of Emergency Medicine, St Michael's Hospital, Toronto, ON; and the „Department of Medicine, University of Toronto.

Correspondence to: Dr. Andrew Petrosoniak, St. Michael's Hospital, Department of Emergency Medicine, 30 Bond Street, Toronto, ON M5B 1W8; Email: petro82@gmail.com

(c) Canadian Association of Emergency Physicians 
A

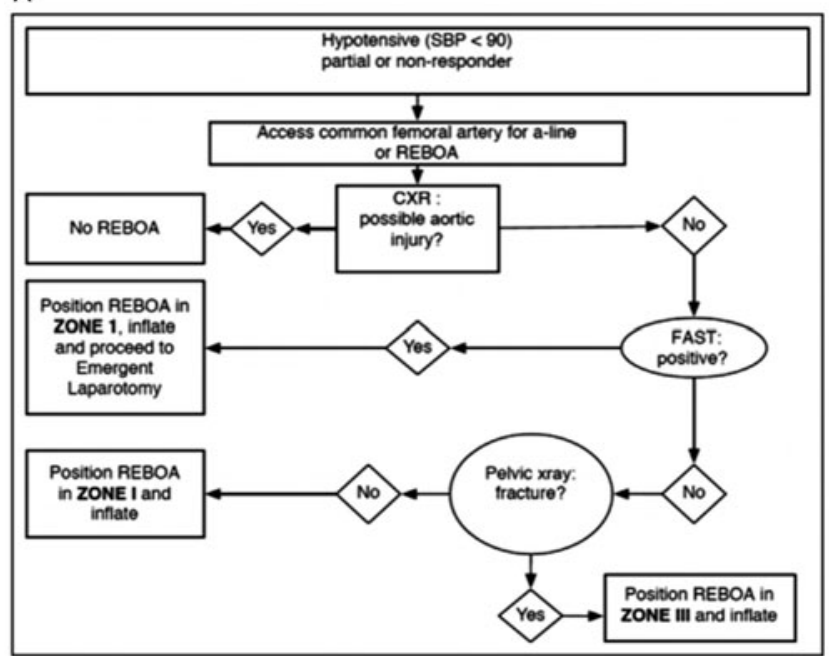

B

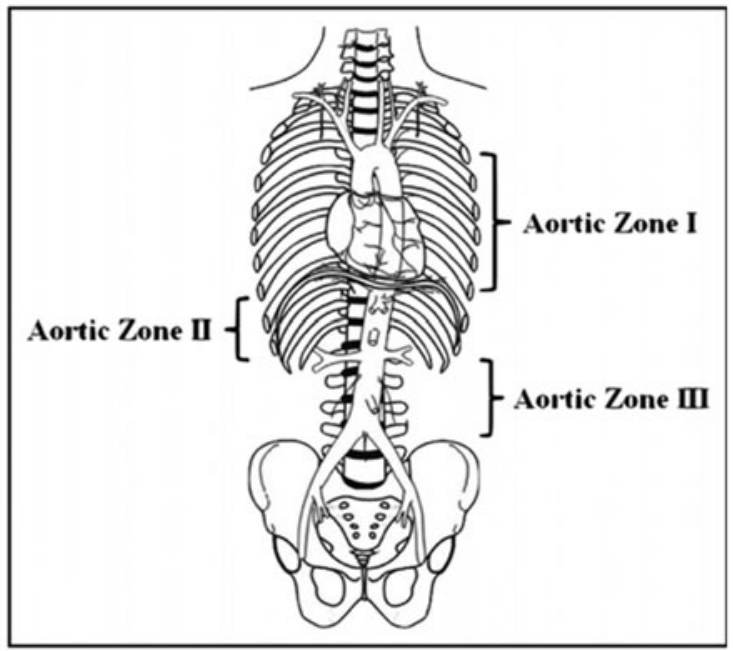

Figure 1. REBOA placement algorithm (A). Aortic zones of occlusion (B), adapted from Stannard, Eliason, and Rasmussen. ${ }^{9}$ Zone 1 between the left subclavian artery and celiac artery is the recommended location for suspected intra-abdominal hemorrhage; Zone 2 between the celiac arteries and renal arteries is not a recommended placement location; and Zone 3, from the lowest renal artery to the aortic bifurcation, is the recommended location for suspected pelvic hemorrhage. Following the decision to proceed with REBOA placement, the clinician must determine where to position the occlusive balloon (Figure 1A). In this case, the patient had a positive FAST, and following the algorithm in Figure 1A, we placed the device in Zone 1.

Our massive transfusion protocol was activated, and the patient received six additional units of pRBCs, three units of FFP, one additional gram of TXA, and one pool of platelets upon arrival at our centre. His sBP did not increase above $60 \mathrm{~mm} \mathrm{Hg}$; however, he continued to demonstrate neurologic function with reactive pupils and a GCS of 7T (E2VTM5), localizing the endotracheal tube with both hands.

The TTL and trauma surgeon considered options for this patient, including an urgent transfer to the OR, resuscitative thoracotomy, and/or REBOA. While this patient, who was in profound hemorrhagic shock with a positive FAST exam, had indications for immediate surgery, transfer to the $\mathrm{OR}$ at our institution requires approximately 10 minutes because of the distance and travel by elevator. Given the persistent shock state $(\mathrm{sBP}<$ $60 \mathrm{~mm} \mathrm{Hg}$ ) and concern for cardiac arrest despite substantial resuscitative efforts, the transfer was deemed unsafe. Approximately one hour after the patient arrived at our institution (10:55), as an alternative to resuscitative thoracotomy with aortic cross-clamping, the surgeon-TTL team collaboratively elected to place a Zone 1 REBOA device as a temporizing measure to facilitate transfer to the OR (Figure 1).

While the TTL continued to guide resuscitation, the surgery team obtained central venous access in the right subclavian vein for blood product administration. The
REBOA procedure began at 10:58 with ultrasoundguided puncture of the left common femoral artery, followed by the advancement of a 0.035 inch, $260 \mathrm{~cm}$ guidewire using a modified Seldinger technique. Serial dilation allowed insertion of a $14 \mathrm{Fr}$ vascular access sheath. A Coda $46 \mathrm{~mm}$ balloon catheter (Cook Incorporated, Bloomington, IN, USA) was inserted into the distal thoracic aorta, followed by guidewire removal. The balloon was inflated with sterile saline until the sBP suddenly increased from $60 \mathrm{~mm} \mathrm{Hg}$ to $105 \mathrm{~mm} \mathrm{Hg}$; a total of $7 \mathrm{cc}$ of saline was used for inflation. Shortly after that, the epinephrine infusion was stopped, and the norepinephrine dose was decreased to $0.5 \mathrm{mcg} / \mathrm{kg} / \mathrm{min}$. Seven minutes elapsed from decision to REBOA and successful aorta occlusion.

The patient was transferred urgently to the $\mathrm{OR}$ and underwent a splenectomy, a small bowel resection, and pre-peritoneal pelvic packing. The largest volume of blood and most likely source of persistent hemodynamic instability was felt to be the pelvic injuries. After surgical hemostasis, but while still in the OR, the Coda balloon was deflated by the surgical team, with no change in mean arterial pressure $(60-65 \mathrm{~mm} \mathrm{Hg})$. There was resistance upon attempting to remove the device that may have occurred because the balloon did not fully retract into the introducer sheath. To ensure complete deflation of the balloon and facilitate removal of the 
device, the catheter was transected, severing the lumen communicating with the balloon, and was then successfully removed without resistance.

A temporary abdominal closure was performed because of persistent coagulopathy (international normalised ratio [INR] of 2.25-3.7), followed by immediate pelvic angiography. There was no evidence of injury or REBOA complication in any Zone 1 or 2 vessels. Bleeding from bilateral internal iliac artery injuries required proximal embolization. The left external iliac artery demonstrated absent flow with clinical signs of ischemia including a pulseless and pale left foot two hours after removal of the REBOA catheter. Seven hours after arrival at our institution (and 12 hours postinjury), he was returned to the OR for vascular sheath removal, arteriorrhaphy, left femoral thrombectomy, and four-compartment fasciotomies of the left leg. The patient demonstrated increasing hemodynamic instability and worsening multi-organ dysfunction, prompting a repeat laparotomy. Several unnamed mesenteric vessels at the site of a prior bowel resection were ligated; however, the patient continued to bleed because of profound coagulopathy despite laboratory-guided blood product administration. Unfortunately, the patient died of his injuries 18 hours after his arrival at our institution. This report was approved by St. Michael's Hospital Research Ethics Board (18-081).

\section{DISCUSSION}

Numerous trauma centres worldwide now include ED-REBOA within their algorithm for the management of hemorrhagic shock; however, to our knowledge, this is the first report of a REBOA performed in a Canadian ED. ${ }^{3,4}$ As part of our local quality improvement process, we reviewed this case and identified a number of important lessons related to the clinical logistics and implementation of ED-REBOA that may be valuable for other institutions with little experience applying this technology (Table 1).

\section{REBOA indications and complications}

The established indications for REBOA insertion remain institution specific and may be influenced by local factors of timeliness of access to definitive care. Our institutional policy is slightly more restrictive than published criteria (Table 2), as we perform REBOA only for blunt trauma

\begin{tabular}{|c|c|}
\hline & Insights and reflections \\
\hline Team training & $\begin{array}{l}\text { Deliberate practice for clinicians } \\
\text { performing the procedure } \\
\text { Multi-disciplinary simulation-based } \\
\text { team training to practice set-up, } \\
\text { assistance during device placement, } \\
\text { and time-critical transport for definitive } \\
\text { hemorrhage control }\end{array}$ \\
\hline Kit assembly & $\begin{array}{l}\text { Intuitive design and kit packaging of all } \\
\text { necessary equipment for femoral } \\
\text { arterial line and REBOA placement }\end{array}$ \\
\hline $\begin{array}{l}\text { Collaboration with } \\
\text { vascular surgery }\end{array}$ & $\begin{array}{l}\text { Develop institutional policy along with the } \\
\text { vascular surgery department }\end{array}$ \\
\hline $\begin{array}{l}\text { Indications for REBOA } \\
\text { application }\end{array}$ & $\begin{array}{l}\text { Establish clear indications for REBOA } \\
\text { deployment following evidence-based } \\
\text { guidelines and in keeping with } \\
\text { institutional resources }\end{array}$ \\
\hline $\begin{array}{l}\text { Quality assurance } \\
\text { program }\end{array}$ & $\begin{array}{l}\text { Record and review all REBOA } \\
\text { deployments to monitor key metrics } \\
\text { and potential complications } \\
\text { Use these data to inform protocol and } \\
\text { training adjustments }\end{array}$ \\
\hline
\end{tabular}

patients with either pelvic ring fractures or a positive FAST with an sBP of $<90 \mathrm{~mm} \mathrm{Hg}$ that is refractory to damage control resuscitation. ${ }^{4}$ REBOA contraindications are informed by a combination of expert opinion and clinical experience (Table 2). ${ }^{3}$

Vascular complications remain a significant concern in REBOA application. The requisite large-bore arterial puncture and occlusion, coupled with a frequent need

\begin{tabular}{|c|c|}
\hline Indications ${ }^{8}$ & Contraindications $^{3}$ \\
\hline $\begin{array}{l}\text { 1. Traumatic hemorrhage } \\
\text { below the diaphragm } \\
\text { unresponsive or transiently } \\
\text { responsive to fluids } \\
\text { 2. Traumatic cardiac arrest } \\
\text { because of hemorrhage } \\
\text { below the diaphragm }\end{array}$ & $\begin{array}{l}\text { 1. Inability to achieve vascular } \\
\text { access (e.g., bilateral femoral } \\
\text { artery injuries) } \\
\text { 2. Known or suspected proximal } \\
\text { aorta injury } \\
\text { 3. Inability to achieve definitive } \\
\text { hemostasis (e.g., no surgeon } \\
\text { available) } \\
\text { 4. Pre-existing indications to } \\
\text { proceed to resuscitative } \\
\text { thoracotomy }\end{array}$ \\
\hline
\end{tabular}


for vascular cut-down, if the Seldinger-technique access fails, may result in substantial complications including aortoiliac dissection, rupture, thrombosis, pseudoaneurysm, and distal thromboembolism. ${ }^{1}$ Some authors report as high as a $40 \%$ amputation rate among survivors of REBOA using large-calibre sheaths. ${ }^{5}$ In our patient, unilateral lower extremity ischemia would have represented a significant source of morbidity had the patient survived. A newly developed REBOA balloon device (Prytime Medical, Lakewood, CO, USA) that is introduced through a 7 Fr sheath, instead of a 12-14 Fr sheath, may decrease these risks, though this remains an outstanding concern of the technique. While a smaller diameter device would likely decrease some complications, we are reminded that REBOA is an invasive technique, with significant risks and potential morbidity.

\section{Equipment and logistical considerations}

The REBOA kit, which has been stocked in our trauma bay for three years, consists of three mini bundles within a single package assembled at our institution (Supplementary Table 1). This intuitive all-in-one packaging system facilitated rapid deployment by our team, who had never placed the device previously. Importantly, we achieved aortic occlusion within seven minutes that was only marginally slower than the reported time-to-occlusion in a large case series (3.5-6 minutes) at centres where REBOA is an established practice. ${ }^{1,6}$ Use of a welldesigned equipment bundle may reduce unnecessary delays related to missing or poorly organized equipment.

Despite the clear visibility of our REBOA kit within our trauma bay, many team members are unfamiliar with REBOA indications and management principles. We identified a clear need for team-based education related to equipment application, physiologic monitoring, and post-inflation transfer. As Canadian trauma surgeons typically do not manage peripheral vascular injuries, an institutional protocol including vascular surgery stakeholders may simplify post-insertion management plans, and this is the guideline recommended. ${ }^{1}$ Infrequent use of REBOA requires multi-disciplinary team training to optimize outcomes. ${ }^{7}$ We estimate, based on a review of our trauma registry, that one to two patients a month would receive REBOA. This case represented the first REBOA application by the trauma surgeon; however, he had extensive experience with the device in animal model research studies.

\section{CONCLUSIONS}

We report the first case of a REBOA placed in a Canadian ED. The application of this endovascular technology produced a dramatic hemodynamic response that facilitated transport from our trauma bay to definitive hemorrhage control, though ultimately the patient did succumb to his injuries and traumatic coagulopathy. The decision to proceed with REBOA warrants careful consideration including local expertise and access to definitive therapies, given the potential for serious complications. Future efforts should focus on identifying patients who would and would not benefit from this invasive procedure.

Acknowledgements: The authors would like to thank Melissa McGowan and Amanda McFarlane for their logistical expertise and the members of the trauma team involved in this case.

Financial support: None.

Competing interests: None.

Supplementary material: The supplementary material for this article can be found at https://doi.org/10.1017/cem.2018.476.

\section{REFERENCES}

1. Brenner M, Inaba K, Aiolfi A, et al. Resuscitative endovascular balloon occlusion of the aorta and resuscitative thoracotomy in select patients with hemorrhagic shock: early results from the american association for the surgery of trauma's aortic occlusion in resuscitation for trauma and acute care surgery registry. 7 Am Coll Surg 2018;226(5):730-40.

2. Rice T, Ahmed N, Rezende-Neto J, et al. Proof of principle in a Canadian level I trauma centre: the use of resuscitative endovascular balloon occlusion of the aorta (REBOA). Can $\mathcal{F}$ Surg 2016;59(3 Suppl 1).

3. Moore LJ, Martin CD, Harvin JA, Wade CE, Holcomb JB. Resuscitative endovascular balloon occlusion of the aorta for control of noncompressible truncal hemorrhage in the abdomen and pelvis. Am 7 Surg 2016;212(6):1222-30.

4. Sato R, Kuriyama A, Takaesu R, et al. Resuscitative endovascular balloon occlusion of the aorta performed by emergency physicians for traumatic hemorrhagic shock: a case series from Japanese emergency rooms. Crit Care 2018;22(1):103.

5. Saito N, Matsumoto H, Yagi T, et al. Evaluation of the safety and feasibility of resuscitative endovascular balloon occlusion of the aorta. 7 Trauma Acute Care Surg 2015;78 (5):897-903.

6. Brenner ML, Moore LJ, DuBose JJ, et al. A clinical series of resuscitative endovascular balloon occlusion of the aorta for hemorrhage control and resuscitation. 7 Trauma Acute Care Surg 2013;75(3):506-11.

7. Davidson AJ, Russo RM, Reva VA, et al. The pitfalls of resuscitative endovascular balloon occlusion of the aorta: risk 
Markus T. Ziesmann et al.

factors and mitigation strategies. 7 Trauma Acute Care Surg 2018;84(1):192-202.

8. Brenner M, Bulger EM, Perina DG, et al. Joint statement from the American College of Surgeons Committee on Trauma (ACS COT) and the American College of Emergency Physicians (ACEP) regarding the clinical use of Resuscitative
Endovascular Balloon Occlusion of the Aorta (REBOA). Trauma Surg Acute Care Open 2018;3(1):e000154.

9. Stannard A, Eliason JL, Rasmussen TE. Resuscitative endovascular balloon occlusion of the aorta (REBOA) as an adjunct for hemorrhagic shock. 7 Trauma 2011;71(6): 1869-72. 